\title{
Multi-region and single-cell sequencing reveal variable genomic heterogeneity in rectal cancer
}

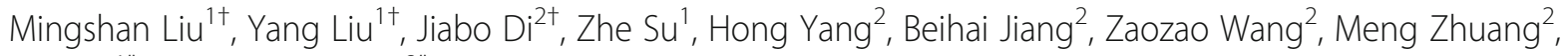
Fan Bai ${ }^{{ }^{*}}$ and Xiangqian $\mathrm{Su}^{2^{*}}$

\begin{abstract}
Background: Colorectal cancer is a heterogeneous group of malignancies with complex molecular subtypes. While colon cancer has been widely investigated, studies on rectal cancer are very limited. Here, we performed multi-region whole-exome sequencing and single-cell whole-genome sequencing to examine the genomic intratumor heterogeneity (ITH) of rectal tumors.

Methods: We sequenced nine tumor regions and 88 single cells from two rectal cancer patients with tumors of the same molecular classification and characterized their mutation profiles and somatic copy number alterations (SCNAs) at the multi-region and the single-cell levels.

Results: A variable extent of genomic heterogeneity was observed between the two patients, and the degree of ITH increased when analyzed on the single-cell level. We found that major SCNAs were early events in cancer development and inherited steadily. Single-cell sequencing revealed mutations and SCNAs which were hidden in bulk sequencing. In summary, we studied the ITH of rectal cancer at regional and single-cell resolution and demonstrated that variable heterogeneity existed in two patients. The mutational scenarios and SCNA profiles of two patients with treatment naïve from the same molecular subtype are quite different.

Conclusions: Our results suggest each tumor possesses its own architecture, which may result in different diagnosis, prognosis, and drug responses. Remarkable ITH exists in the two patients we have studied, providing a preliminary impression of ITH in rectal cancer.
\end{abstract}

Keywords: Rectal cancer, Single-cell whole-genome sequencing, Multi-region whole-exome sequencing, Somatic copy number alterations, Intratumor heterogeneity

\section{Background}

Colorectal cancer is highly heterogeneous, and its pathogenesis and molecular classification have been widely investigated [1, 2]. In fact, colon and rectal cancers not only have different clinicopathological features, but also undergo different molecular paths of tumorigenesis [3].

\footnotetext{
* Correspondence: fbai@pku.edu.cn; suxiangqian@bjmu.edu.cn

${ }^{\dagger}$ Equal contributors

'Biodynamics Optical Imaging Center (BIOPIC), School of Life Sciences, Peking University, No. 5 Yiheyuan Road, Haidian District, Beijing 100871, China

${ }^{2}$ Key Laboratory of Carcinogenesis and Translational Research (Ministry of Education), Department of Gastrointestinal Surgery IV, Peking University Cancer Hospital \& Institute, 52 Fucheng Road, Haidian District, Beijing 100142, China
}

Tumor heterogeneity, a notable feature of cancer, has recently been studied in breast cancer [4], esophageal cancer [5], renal cancer [6, 7] and lung cancer $[8,9]$ through multi-region sequencing of tumor masses. Intratumor heterogeneity (ITH) and branched evolution were commonly observed, and the complexity of the tumor tissue composition was beyond expectation. However, tumor heterogeneity of colorectal cancer, especially rectal cancer, was less investigated.

ITH can be assessed by single-cell sequencing, as recent progress in single-cell genome sequencing has allowed quantitative characterization of both single nucleotide variations (SNVs) and somatic copy number alterations (SCNAs) in individual tumor cells. For instance, single-cell 
sequencing of individual circulating tumor cells (CTCs) revealed reproducible SCNA patterns in CTCs from the same patient and identified pertinent cancer mutations [10]. Single-cell sequencing of a large number of breast tumor cells [11-13] revealed punctuated evolution of SCNAs during tumor development. In addition, single-cell exome sequencing analysis of a case of colon cancer revealed a biclonal tumor origin and proved low-prevalence mutations could also play a role in tumorigenesis [14]. Nevertheless, the ITH of rectal cancer has not been well studied by single-cell sequencing.

In the current study, we performed multi-region whole-exome sequencing (WES) and single-cell wholegenome sequencing (WGS) to evaluate the ITH of two rectal tumors. The SCNAs and mutations were exquisitely identified from multi-region to single-cell level. We found that the extent of ITH in the two patients was variable, and the degree of heterogeneity increased when analyzed on the single-cell level.

\section{Methods}

\section{Sample collection and single cell preparation}

We obtained two fresh primary rectal tumors from patients who underwent primary tumor resection at the Department of Gastrointestinal Surgery IV, Peking University Cancer Hospital \& Institute. None of them received radiotherapy or chemotherapy before surgery. The clinicopathological characteristics of the two patients are listed in Additional file 1: Table S1. Sections were collected from different regions of tumors immediately after surgical removal. To obtain single-cell suspensions, each region was washed, minced with sterile blades into small pieces, and dissociated by incubation in DMEM containing collagenase type IA $(50 \mu \mathrm{g} / \mathrm{mL}$; Sigma-Aldrich Co. LLC, US), hyaluronidase $(20 \mu \mathrm{g} / \mu \mathrm{L}$; Sigma-Aldrich Co. LLC, US), and antibiotics/antimyotics for $1 \mathrm{~h}$ at $37^{\circ} \mathrm{C}$. After digestion, cells were filtered through a $70 \mu \mathrm{m}$ cell strainer (BD Falcon ${ }^{\mathrm{Tm}}$, US), and erythrocytes were removed by treatment with $\mathrm{NH}_{4} \mathrm{Cl} / \mathrm{EDTA}$. Cells were then cryopreserved in liquid nitrogen. Peripheral blood from each patient was collected and stored at $-20{ }^{\circ} \mathrm{C}$.

\section{The fluorescent activated cell sorting (FACS) and single-cell isolation}

To isolate single tumor cells, cryopreserved cells were thawed and stained with combinations of the following reagents: anti-EpCAM Alexa Fluor 488 (eBioscience, US), and lineage-specific antibodies, including anti-CD45-PE (BD Pharmingen $^{\mathrm{TM}}$, US), anti-CD235a-PE (BD Pharmingen ${ }^{\mathrm{TM}}$, US), anti-CD140b-PE (BD Pharmingen ${ }^{\mathrm{TM}}$, US), and antiCD31-PE (BD Pharmingen ${ }^{\mathrm{Ts}}$, US). To discriminate viable cells, 7-Amino-Actinomycin D (7-AAD, BD Pharmingen ${ }^{\mathrm{TM}}$, US) was labeled 5-10 min before sorting. Single tumor cells were sorted based on $7-\mathrm{AAD}^{-}$lineage ${ }^{-} \mathrm{EpCAM}^{\text {high }}$ by $\mathrm{BD}$
FACS Aria III (BD Biosciences, US). Individual tumor cells were verified under the fluorescence microscopy (Nikon Eclipse Ti, Japan) and separated by mouth pipetting. Isolated single cells were then lysed.

\section{Whole-exome library preparation and sequencing}

We used the QIAamp Micro DNA kit (QIAGEN, US) to extract genomic DNA from the single-cell suspension derived from sections and matched blood, and the concentrations were measured by Qubit 2.0 fluorometer (Invitrogen, US). Total gDNA ( 600 ng) was sheared into fragments $(\sim 180-280 \mathrm{bp})$ by the Covaris system (Covaris, US). Libraries were generated using the Agilent SureSelect Human All Exon V6 kit (Agilent Technologies, US) following the manufacturer's recommendations, and index codes were added to each sample. The products were sequenced with Illumina Hiseq4000 $2 \times 150$-bp PE reads at $\sim 100 \times$ depth.

\section{Whole-genome library preparation and sequencing}

After lysis, single cells were amplified by the multiple annealing and looping-based amplification cycles (MALBAC) method [15]. The cells passed the quantitative PCR (qPCR) quality control [10] were used for next-generation sequencing (Bio-Rad, US). DNA ( 600 ng) from each single cell and gDNA $(\sim 500 \mathrm{ng})$ from tumor tissue was sheared into $\sim 300$ bp fragments by the Covaris system (Covaris, US), and the indexed libraries were prepared with the NEBNext Ultra DNA Library Prep Kit for Illumina (New England Biolabs, US). The products were then sequenced with Illumina HiseqXTen $2 \times 150$-bp PE reads at $\sim 0.3 \times$ depth.

\section{Analysis of WES data}

The reads were aligned to the human reference genome (hg19, USCC) with the Burrows-Wheeler Aligner [16]. The aligned BAM files were sorted and merged with Samtools 0.1.19 [17]. First, we applied two software, the Genome Analysis Toolkit (GATK 1.6) [18] and multiSNV [19], to identify mutations in multi-region WES. The INDELs and SNVs were identified with GATK 1.6 [18] based on dbSNP 135 (www.ncbi.nlm.nih.gov/projects/ $\mathrm{SNP} /$ ), and the duplicates were removed with Picard-tools 1.76 (http://Picard.Sourceforge.net). The functional effect of variants was annotated using SNPEFF3.0 [20]. Then, the SNVs and INDELs (insertion and deletion) were filtered out based on previous criteria [21] using the Catalog of Somatic Mutations in Cancer (COSMIC) database v61. We manually filtered out tumor mutations with base quality of lower than 30 and distance between two mutations of lower than $15 \mathrm{bp}$. Germline mutations were removed by comparing the tumor data to matched blood data. Next, we input the aligned BAM files into multiSNV [19] to call the SNVs. Germline SNPs were removed by comparing the tumor data to matched 
blood data. After that, low quality SNPs were filtered and the functional effect of variants was annotated using SNPEFF3.0 [20]. Shared SNVs of each region by the two software were used for subsequent analysis. Additionally, to reduce the false negative rate, we had manually assessed the SNVs which had low allelic frequency in samples. Some SNVs existed in two or more samples of one patient, but were detected by either software in only one sample. Then we would screened manually in these SNVs, of which if variant allelic frequency (VAF) in samples was more than 0.2 we would put them back into our SNV list. Eventually, we added the INDELs identified by GATK into the shared SNV list to get the final mutations for further analysis.

Phylogenetic trees were constructed by MEGA5 with maximum likelihood method [22], and potential driver mutations were labelled on branches with Adobe Illustrator. The purities and SCNA profiles of multiple tumor regions from one patient were estimated with the Sequenza $R$ package 2.1.1 [23].

\section{The SCNA profiles of the tumor regions}

The libraries of tumor regions and match blood constructed with gDNA were performed WGS. The clean data was aligned to human reference genome (hg19, UCSC) with the Burrows-Wheeler Aligner [16]. After that, we sorted and merged each sample with Samtools 0.1.19 [17]. To visualize the SCNA profiles of WGS, we sorted the whole genome into $500 \mathrm{~Kb}$ bins (on average), and then used matched blood as control to remove noises. Finally, the depth of each bin of tumor regions was plotted along the order of the chromosomes.

\section{The single-cell SCNA profiling}

The single-cell SCNA profiles were identified using previously described methods $[10,15]$. The reads were aligned to human reference genome (hg19, UCSC) with the Burrows-Wheeler Aligner [16] and then sorted and merged with Samtools 0.1.19 [17]. The whole genome was sorted into $500 \mathrm{~Kb}$ bins (on average), and the depth of each bin was determined by the hidden Markov model normalized with the method control [10].

\section{Single-cell WGS analyses}

The median of the absolute values of all pairwise differences (MAPD) was used to assess the quality of the single-cell data [24]. The MAPD scores of the 88 cells were less than 0.25 , and all of them passed the quality control. The clustered heat map of the large-scale copy number profiles was generated by the Euclidean distance and ward.D method and visualized by the heatmap. 2 function in the gplots package. The principle component analysis (PCA) was performed with the prcomp function in the stats package. Partition around medoids (PAM) clustering was performed using the pamk function in the fpc package. The consensus copy number profiles of multiple regions were inferred from single tumor cells based on the median value of each bin.

\section{Identification of subclonal SCNAs}

The subclonal SCNAs of single cells were identified by PCA using the FactoMineR package based on the depth of each bin (each patient had 6037 bins at $500 \mathrm{~Kb}$ ) and were visualized with the gplots package. We integrated the bins of single tumor cells from each patient into one matrix and filtered out the bins with all elements equal to zero. Each included bin had at least three elements greater than zero. Then, we set the variance of each bin to greater than 0.5 to obtain subclonal SCNAs with high disparities. There were 116 and 1637 bins containing subclonal SCNAs collected from PC1 to PC6 for patients 1 and 2, respectively. After that, we manually selected subclonal SCNAs larger than 1.5 Mb (63 and 806 bins for patients 1 and 2, respectively), and visualized the results with clustered heat maps.

\section{Single-cell mutation validation}

The mutations identified in the multi-region WES were validated in single cells by Sanger sequencing (Ruibiotech, China) using $20 \mathrm{ng}$ of the MALBAC products as DNA templates. The PCR was performed with OneTaq Hot Start Quick-Load 2× Master Mix (New England Biolabs, US). The thermal profile was $94{ }^{\circ} \mathrm{C}$ for $60 \mathrm{~s} ; 35$ cycles of $94{ }^{\circ} \mathrm{C}$ for $25 \mathrm{~s}, 58{ }^{\circ} \mathrm{C}$ for $30 \mathrm{~s}$, and $68{ }^{\circ} \mathrm{C} 40 \mathrm{~s}$; and $68{ }^{\circ} \mathrm{C}$ for 5 mins. The primers used are listed in Additional file 1: Table S2.

We used ploidy status and ubiquitous mutations to distinguish somatic diploid cells and tumor cells. We used five or six nonsynonymous ubiquitous mutations which were identified in multi-region WES as candidate mutations to exclude somatic diploid cells (Additional file 1: Table S3). A single cell was considered to be somatic diploid cells if the candidate mutations were validated as wildtype by Sanger sequencing, while tumor cells had SCNAs and mutations. Owing to allelic dropout and imbalanced single-cell amplification, some mutations were undetectable in single cells, but were validated in gDNA of the tumor. As shown in Table S3, the candidate mutations were all validated in the gDNA of the two tumors, but sporadically identified in single cells. There were 15 diploid cells excluded in patient 1 , of which two cells (B1 and C8) containing more than three mutations were excluded in the later analysis, owing to the possibility that they were a mixture of one diploid cell and debris of tumor cells. The number of diploid cells in patient 2 was 13 , and none of the six candidate mutations were validated in them. In total, 26 cells (13 from patient 1 and 13 from patient 2) were confirmed to be somatic diploid cells, and two cells 
(B1 and C8 of patient 1) seemed to be mixtures, which were all excluded in further analysis of tumor cells.

Considering the phylogenetic trees, putative driver mutations in the COSMIC database, disease-associated genes identified by DAVID [25, 26] and possible driver mutations in cancer genome landscape [27], we selected 14 nonsynonymous mutations for each patient and validated the presence of these WES identified mutations in single tumor cells with SCNAs. The single cells with SCNAs were confirmed to be tumor cells if at least four mutations were present.

\section{Results}

\section{Multi-region WES revealed variable genomic heterogeneity}

To depict the genomic heterogeneity of rectal cancer, multi-region WES was performed to determine the mutation distribution and SCNAs profiles in the two rectal primary tumors. The two fresh primary rectal tumors were of the same molecular subtype [28], which was microsatellite stable, chromosomal instable (referring to SCNAs here), and/or mutant TP53 with wildtype KRAS and PIK3CA (Additional file 1: Table S1). To obtain mutational profiles, we carried out WES on multiple regions and matched blood (germline comparator) at $~ 100 \times$ depth (Additional file 1: Table S4). For patient 1, four regions (A to D) were sequenced (Fig. 1a), and 141 nonsynonymous mutations involving 138 genes were detected (Fig. 1b, Additional file 1: Table S5). In the five regions (A to E) of patient 2 (Fig. 1C), 119 nonsynonymous mutations involving 117 genes were identified (Fig. 1d, Additional file 1: Table S5). The mutations were categorized as 'ubiquitous', which were mutations shared by all regions of the tumor, 'shared', which were shared by more than one region but not all regions, and 'private, which were specific to a single region. According to the phylogenetic trees which delineated the tumor evolutionary patterns (Fig. 1e and f) and the heat maps of nonsynonymous mutations (Fig. $1 \mathrm{~b}$ and d), analysis of the regional distribution of nonsynonymous mutations revealed more ITH in patient 2 than that in patient 1 . The observation that the mutational heterogeneity of patient 2 was more extensive than that of patient 1 might be due to the fact that the tumor from patient 2 was larger in size and later in stage (Additional file 1: Table S1), implying that a longer disease progression might foster tumor heterogeneity.

As the mutation spectrums showed, $\mathrm{C}>\mathrm{T}$ transitions were prominent in both patients (Fig. $1 \mathrm{~g}$ and $\mathrm{h}$ ). There was no significant difference in the mutation spectrum among the tumor regions of patient 1 ( $\mathrm{X}$-squared test, $p>0.05) . \mathrm{T}>\mathrm{A}$ transversions were detected in patient 2 among the shared and private mutations, especially in region $\mathrm{C}$ (Fisher's exact test, $p<0.05$ ), suggesting that different tumor microenvironment might bring about the differences in mutational profiles [29].

We combined VAF, copy number, and the purity of tumor tissue to analyze the cancer cell fraction of each region as a means to discriminate mutational heterogeneity of each region [30]. As shown in Additional file 1: Fig. S1 and Fig. S2, patient 2 had much more mutations on axes (marked by green and blue) than patient 1 , which were referred to region-specific subclones. Therefore, the multiple regions in patient 2 were more heterogeneous than those of patient 2. Moreover, the mutational scenarios of the two patients were quite different. In patient 1 , mutations in ATM and GNAS, as well as a deletion in the tumor suppressor gene PTEN, likely led to tumorigenesis since they are potential cancer driver genes $[2,27]$. In patient 2 , mutations in TP53, ERBB2 and $A P C$, which were frequently mutated in colorectal tumors and involved in the $\mathrm{WNT} / \beta$-catenin signalling pathway [31], might play important roles in tumorigenesis and could be possible drug targets [32, 33].

Gene mutations are associated with chromosomal instability, a consequence of which is SCNAs [34], and the interactions of these two events facilitate tumor progression. We performed WGS on multiple tumor regions and matched blood at $\sim 0.3 \times$ depth to depict SCNA profiles of each tumor region. The SCNA profiles of the tumor regions for each patient were found to be very similar (mean Pearson correlation coefficient of patient 1 and patient 2 was 0.9713 and 0.9822 , respectively) and highly reproducible (Fig. 1i). The genomes of both patients had gains at chr20q and losses at chr18q, which were accordant with the previously reported frequent copy number changes in colorectal cancer [35]. In addition, we observed common SCNA gains in these two patients at chr1q21-23, chr3q27-28, chr5q32-35, chr6p21, chr8q23-24, chr16p11 and chr17q25, as well as SCNA losses at chr1p22 and chr9q12. Patient 1 had losses at chrX, while patient 2 had gains at chrX. Given that the WGS was performed at a low depth of coverage, to improve the resolution of more focal events, we analyzed SCNA profiles with the WES data eliminating the contamination caused by diploid cells by using Sequenza. The SCNA profiles of the tumor regions in patient 1 also seemed to be similar, while those of certain regions in patient 2 were obviously distinguishable at chr3q and chr8p among the five regions (Additional file 1: Fig. S3). Collectively, these data indicate that the SCNA profiles of the tumor cells in patient 2 were more heterogeneous, and multi-region WES was not sufficient to fully represent the full scenarios of the SCNA profiles.

\section{Single-cell sequencing showed SCNA-based subpopulations}

We performed single-cell WGS to access the ITH of each region at the single-cell level. Tumor cells were 

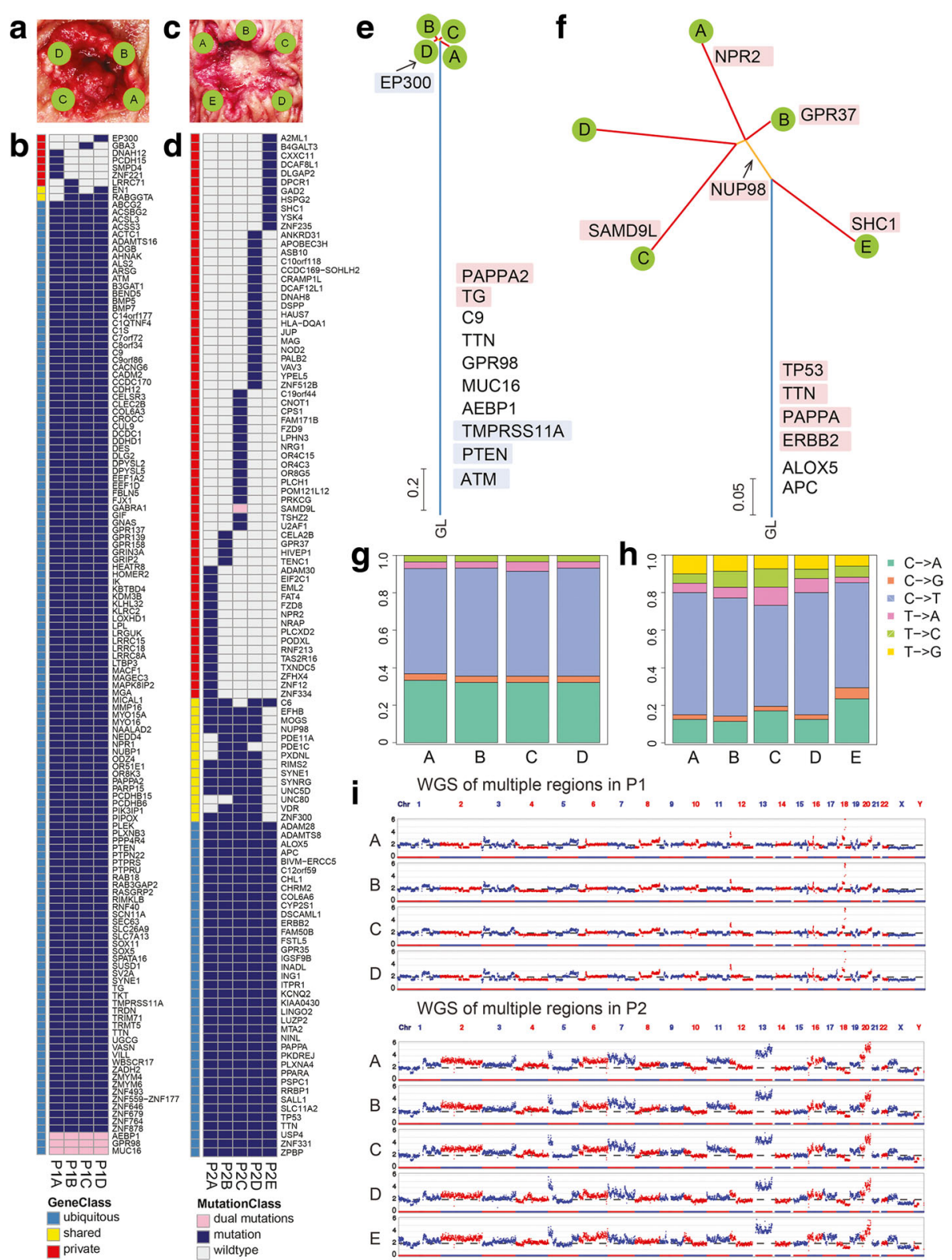

WGS of multiple regions in P1

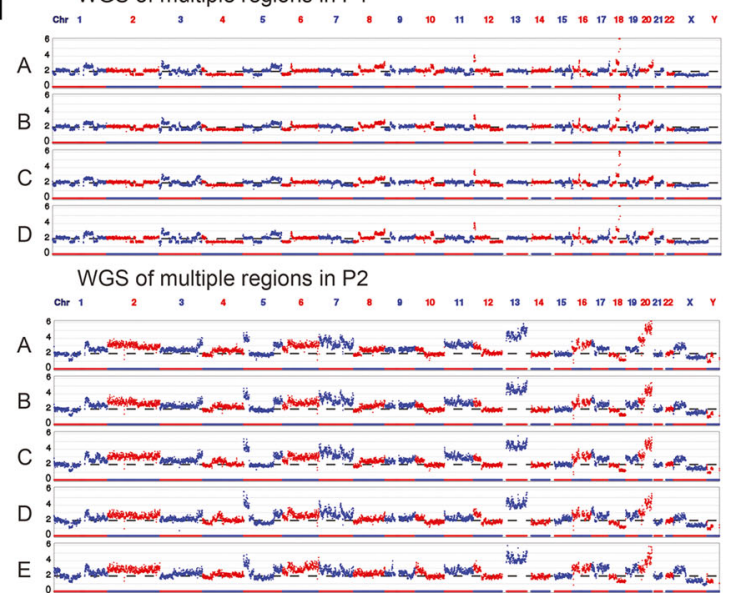

Fig. 1 Multi-region WES revealed variable genomic heterogeneity in two rectal tumors. a The multiple regions of patient 1 divided by physical distance. $\mathbf{b}$ The distribution of nonsynonymous mutations in multiple regions of patient 1 . The blue and the grey in heat map presented the mutations and the absences, respectively. The pink in heat map means this gene had two separate independent mutations. The color bars next to the heat map indicate classification of mutations according to whether they are ubiquitous, shared by some tumor regions but not all, or unique to the region (private). $\mathbf{c}$ The multiple regions of patient 2 divided by physical distance. $\mathbf{d}$ The distribution of nonsynonymous mutations in multiple regions of patient 2. e The phylogenetic tree of patient 1 deduced from multi-region WES. The blue trunk, yellow branches and red leaves represented the clonal, the subclonal and the private mutations, respectively. The red, the white and the blue background of mutations meant the gain $(>2 \mathrm{~N})$, normal $(\sim 2 \mathrm{~N})$ and loss ( $<2 \mathrm{~N})$ of copy number, respectively. The distance of the branch was based on similar probability between samples. $\mathbf{f}$ The phylogenetic tree of patient 2 deduced from multi-region WES. $\mathbf{g}$ The mutation spectrum of multiple regions in patient $1 . \mathbf{h}$ The mutation spectrum of multiple regions in patient 2. i The copy number profiles of multiple regions and blood in patient 1 and patient 2. The SCNAs of genomic DNA from multiple regions (blue) and matched blood (red) detected by whole-genome sequencing was visualized by Circos. P1: patient1; P2: patient 2

sorted by FACS based on the 7-AAD ${ }^{-}$Lineage ${ }^{-} \mathrm{EpCAM}^{\text {high }}$ biomarker combination [36] and then single cells were picked up by micropipetting under microscope. Genomic DNA of each cell was amplified using MALBAC [15], an outstanding whole genome amplification method that allows accurate detection of SCNAs and mutations from single cells $[37,38]$. The SCNA profile of each cell was plotted using previously established protocols $[10,15]$. In 
total, 40 single cells of patient 1 (ten single cells for each region) and 48 single cells of patient 2 (eight single cells for region $\mathrm{D}$ and ten single cells for the other regions) passed the quality control and were subjected to single-cell WGS. Hierarchical clustering showed that the single cells of each patient were divided into two subpopulations, diploid cells and cells with SCNAs (Fig. 2a). PAM clustering [39] was applied to quantify the number of clusters, which also supported the results (Additional file 1: Fig. S4).

We then analyzed the presence of the WES detected mutations in each single cell, and the procedure of normal and tumor cell validation was shown in Fig. 2b. There was a possibility that even after the $7-\mathrm{AAD}^{-}$Lineage ${ }^{-} \mathrm{EpCAM}^{\text {high }}$ enrichment, there were still a few normal stroma cells mixed in the tumor cell population, and these diploid cells were ruled out in the validation procedure (Additional file 1: Table S3). In single tumor cells with SCNAs, we selected a set of mutations identified by multi-region WES and assessed their presence by targeted PCR and Sanger sequencing to exclude the calling of false-positive SNVs inherited from single-cell whole-genome amplification. After validating mutations by Sanger sequencing, we were able to confirm 24 out of 40 cells from patient 1 (Fig. 2c, six for region A, five for region $\mathrm{B}$, five for region $\mathrm{C}$, and eight for region D, Additional file 1: Table S6) and 35 out of 48 cells from patient 2 (Fig. 2d, six for region A, nine for region $B$, eight for region $C$, six for region $D$, and six for region E, Additional file 1: Table S6) as tumor cells with genomes that acquired SCNAs and possessed cancerassociated mutations simultaneously. Of special note, the mutation in PDE11A gene was 'shared' mutation by regions $\mathrm{B}, \mathrm{C}$ and $\mathrm{D}$ in patient 2 (Fig. $1 \mathrm{~b}$ ). However, we found that it also existed in a single cells (A1 and E4, Fig. 2d) in regions A and E, suggesting that the ITH was more extensive on the single-cell level, and the depth $(\sim 100 x)$ of the WES used in the multi-region WES was insufficient to capture all of the low-frequency mutations present in minor subclones.

Single-cell sequencing revealed de novo focal SCNAs that were hidden in the bulk sequencing

After excluding all the diploid cells and one cell doublet (single cell D2 of patient 1) from further analyses, clustering analyses based on large-scale SCNA profiles showed that
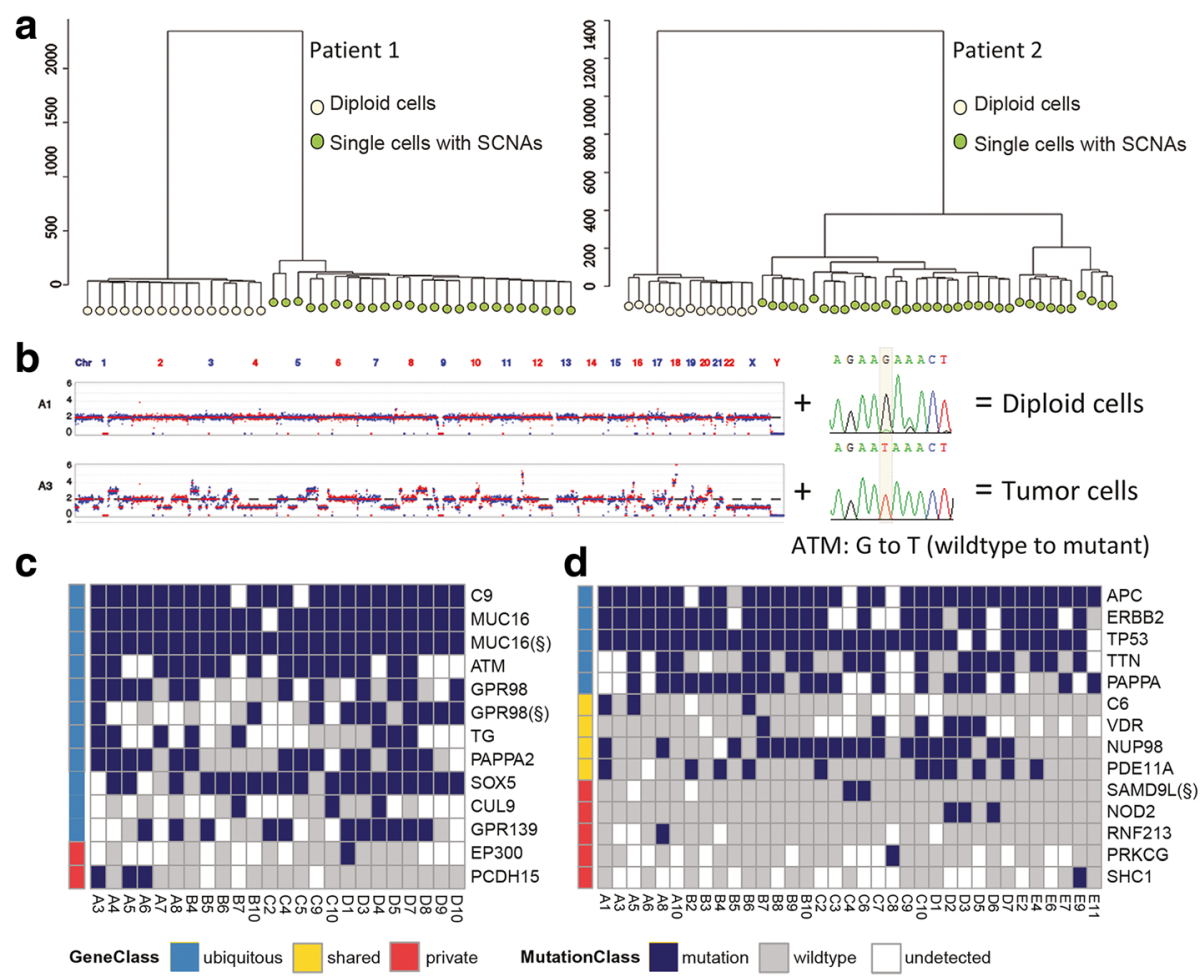

Fig. 2 Single-cell sequencing showed SCNA-based subpopulations within two rectal tumors. a Cluster analysis of single cells of each patient based on copy number profiles. The cluster was constructed by Euclidean distance and ward.D method. The yellow and the green represented diploid cells and tumor cells with SCNAs, respectively. $\mathbf{b}$ The procedure to distinguish between diploid normal and tumor cells. Diploid cells without mutations were considered to be normal cells, while cells with both SCNAs and mutations were considered as tumor cells. c The mutations validated in single tumor cells of patient 1 by Sanger sequencing. The blue, the grey and the white presented the mutations, the absence of mutations and the undetected by PCR, respectively. (§) represented this gene had two separate independent mutations (d) The mutations validated in single tumor cells of patient 2 by Sanger sequencing. SAMD9L(\$) had two base substitution TC to AA at chr7: 92,763,288-92,763,289 
there was one population in patient 1 (Fig. 3a), whereas two subpopulations were detected in patient 2 (Fig. 3b). PAM clustering [39] also supported two subpopulations of patient 2 (Additional file 1: Fig. S4).

We further analyzed the single cell SCNA data with PCA. The subclonal SCNAs of single cells were identified by PCA based on the depth of each bin. The subclonal
SCNAs of more than $1.5 \mathrm{Mb}$ in patient 1 (63 bins) were visualized with a clustered heatmap (Fig. 3c, Additional file 1: Fig. S5). In stark contrast to the large-scale copy number-based clustering, single cells of patient 1 were clustered into two groups based on subclonal SCNAs (>1.5 Mb), supported by PAM clustering [39] which also quantified two clusters (Additional file 1:
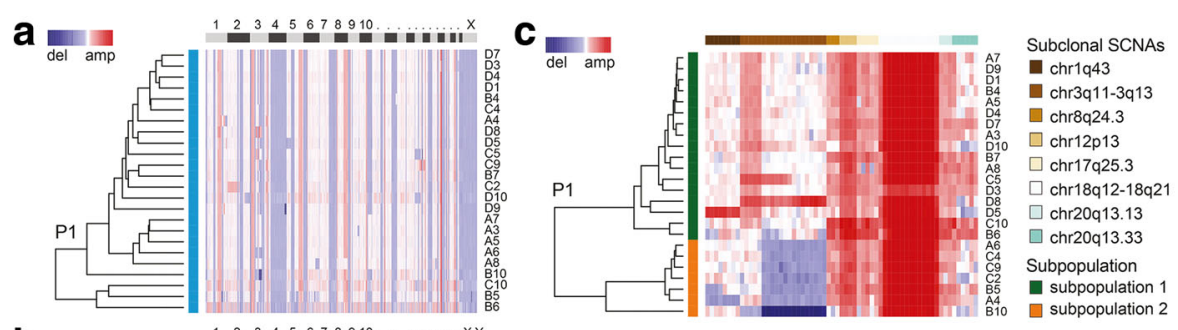

$\mathbf{b}_{\text {del amp }}$
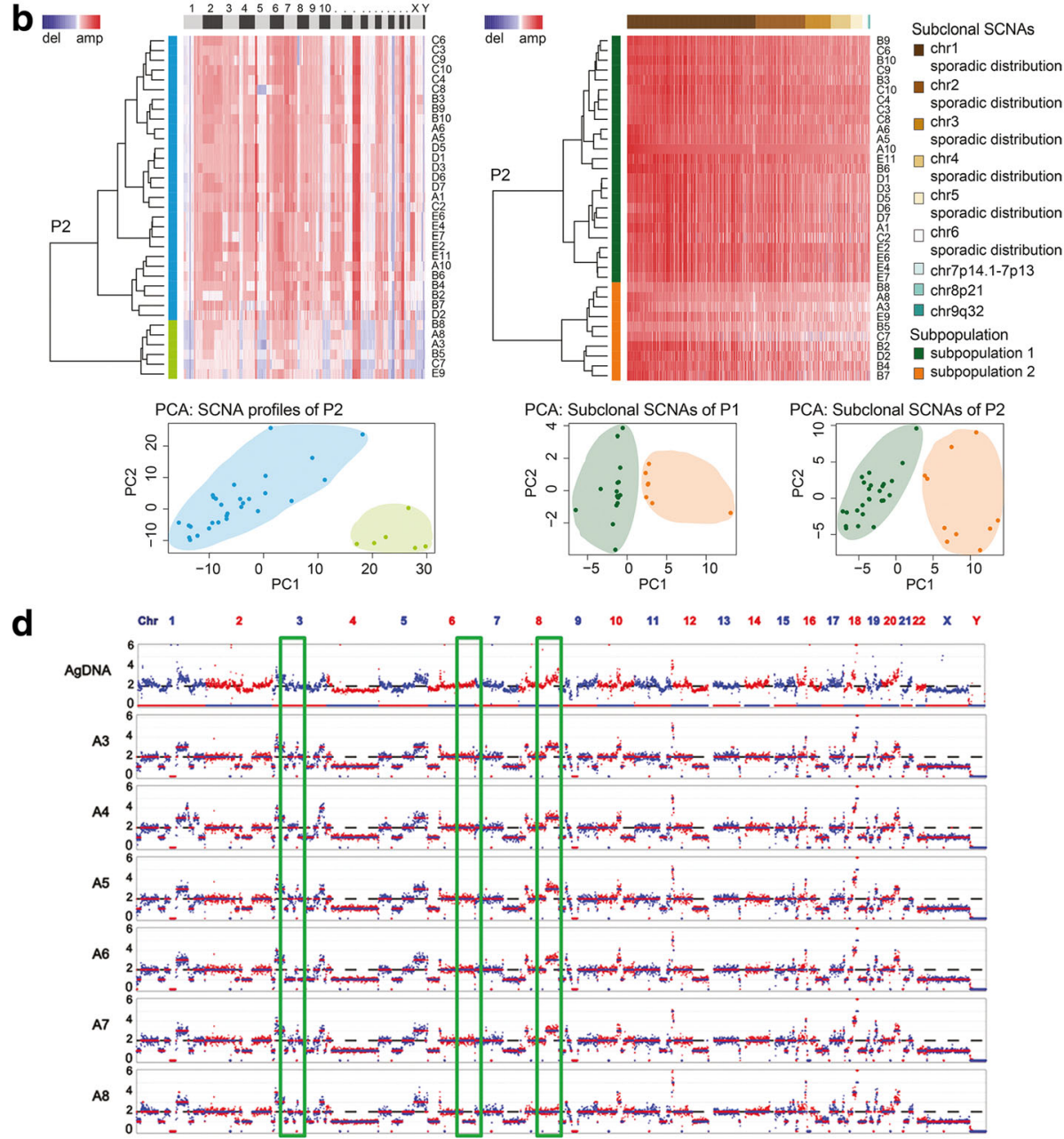

Fig. 3 Single-cell sequencing showed more subtle differences than multi-region WES. a Clustered heatmap of 24 single tumor cells with SCNA profiles in patient 1 based on Euclidean distance and ward.D method. The $\mathrm{x}$ axis was plotted by chromosomes from chr 1 to chrX $Y$ and the $y$ axis was the population labeled by blue. $\mathbf{b}$ Clustered heat map and PCA of 35 single tumor cells of patient 2 based on SCNA profiles. Single tumor cells were grouped into two clusters. The $x$ axis was plotted by chromosomes from chr1 to $c h r X Y$ and the $y$ axis was subpopulations labeled by blue and green. c Subclonal SCNAs of patients 1 and 2 divided single tumor cells into two subpopulations, which was in accordance with two clusters identified by PCA. The chromosomes (columns) where subclonal SCNAs more than $1.5 \mathrm{Mb}$ located was showed in colors. The two subpopulations (rows) were labeled in colors. $\mathbf{d}$ Single tumor cells showed more differences in regional level than gDNA in reigon A of patient 1. P1: patient1; P2: patient 2 
Fig. S4). The subclonal SCNAs of patient 2 were more extensive and complicated (1674/6037 bins before manual selection), which might be related with the advanced stage. Based on the large-scale copy number-based clustering, the PCA of patient 2 confirmed the existence of two subpopulations (Fig. 3b). The single tumor cells of patient 2 were also clustered into two groups based on subclonal SCNAs (806 bins), though the proportion of two subpopulation altered from 29:6 to 25:10, meaning that the preponderant subpopulation based on the large-scale copy number-based clustering might divided into two subclones because of subclonal SCNAs $(29=25+4)$ in the future (Fig. 3c, Additional file 1: Fig. S5). The PAM results [39] also supported two clusters existed (Additional file 1: Fig. S4). These results implied that single tumor cells had different fitness advantages owing to subclonal SCNAs, and could possibly form more subpopulations at a later stage during tumor progression.

The SCNA profiles of genomic DNA extracted from multiple regions were distorted by the presence of somatic diploid cells, whereas the profiles obtained by the sequencing of single tumor cells likely revealed the true differences within the bulk tumor. Therefore, single-cell sequencing is necessary to precisely determine the true number of different subclones within a tumor cell population [40]. For instance, variable SCNAs in certain chromosomal regions in single tumor cells were hidden in the bulk gDNA in region A of patient 1 (Fig. 3d). The frequencies of the two subpopulations based on SCNA profiles in patient 2 were 17\% (6/35) and 83\% (29/35). The SCNA-based subclonal frequencies of patient 2 might explain the regional differences observed in the multiregion WES (Additional file 1: Fig. S3), which arose from the proportions of the two subpopulations in each region.

\section{Differences between the two patients}

We evaluated the ITH of two rectal cancer patients at the multi-region and single-cell levels. Each patient showed unique large-scale copy number patterns (Fig. 4a). Hierarchical clustering and PCA showed that 24 tumor cells of patient 1 and 35 tumor cells of patient 2 were obviously grouped into two populations (Fig. 4b and c). The two patients only had TTN and SYNE1 mutations in common (Fig. 4d), and these genes might play a role in chromosome segregation during mitosis [41] and subcellular spatial organization [42]. Gene Ontology (GO) terms based on biological processes (DAVID 6.7) showed that the mutated genes in patient 1 were clustered in homophilic cell adhesion via plasma membrane adhesion molecules, biological adhesion, and regulation of stem cell differentiation, while the mutated genes in patient 2 were clustered in cell adhesion, neuron

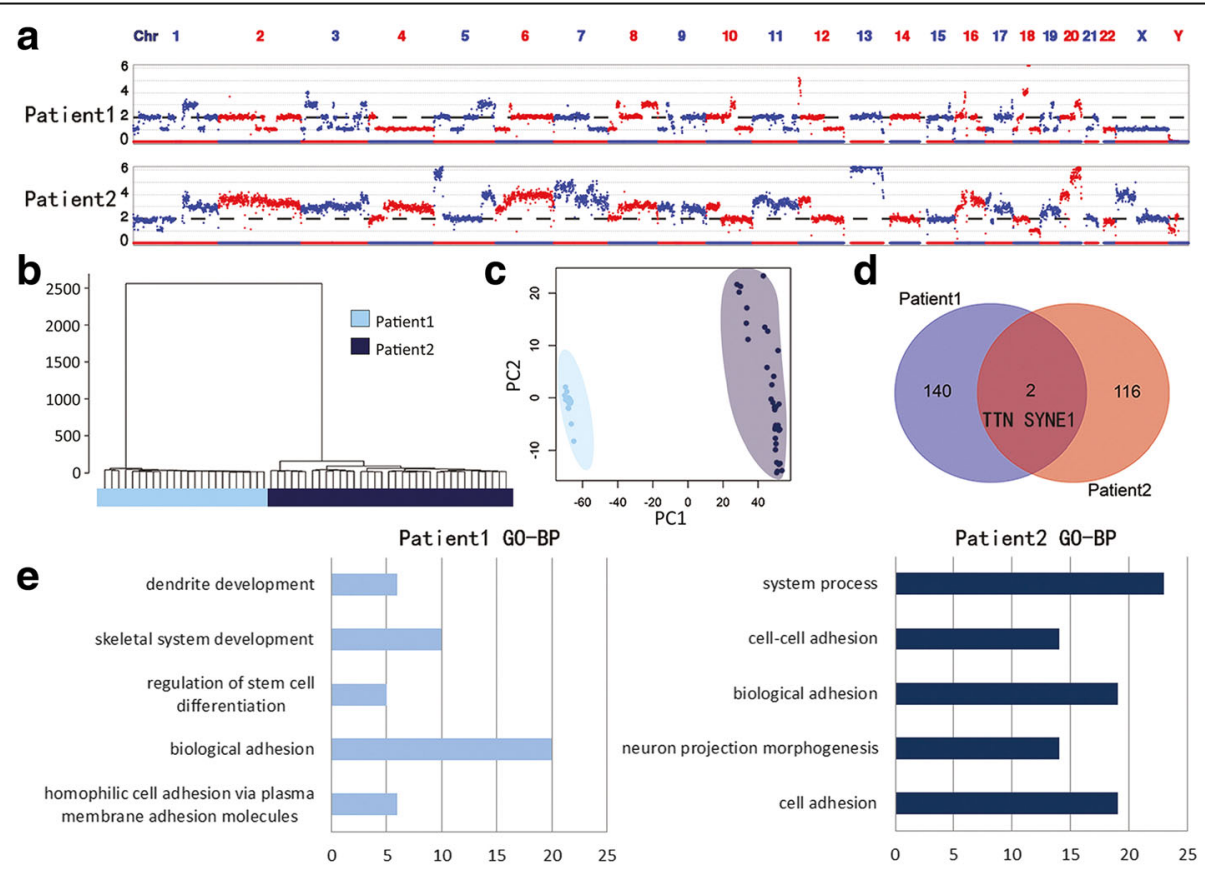

Fig. 4 Individual differences between two patients. a The consensus copy number profiles of two patients. Each patient had a specific individual large-scale copy number pattern. $\mathbf{b}$ The hierarchical clustering using Euclidean distance and ward.D method showed that single tumor cells were grouped into two populations according to two patients. c The PCA showed that single tumor cells were divided into two clusters according to two patients. $\mathbf{d}$ The Venn diagram of mutations from two patients. Two patients merely had TTN and SYNE1 mutated genes in common. e GO-BP analyses of mutated genes in two patients. The top five biological processes of the two patients were quite different and $x$ axis was labeled by the number of mutated genes involved in each process, $p<0.05$ 
projection morphogenesis, and biological adhesion (Fig. 4e). In a word, the copy number profiles and mutational scenarios of the two patients were quite different, suggesting the necessity of personalized medicine in clinical therapy.

\section{Discussion}

In this study, we performed multi-region integrated single-cell sequencing to explore the ITH in two rectal tumors. The large-scale copy number profiles of multiple regions and single tumor cells in each patient appeared to be similar, implying that the majority of chromosomal rearrangements were early events and were inherited clonally and steadily, which was accordant with previous studies on breast cancer [12, 13]. Besides the clonal SCNAs, some subclonal SCNAs were also observed by single-cell sequencing. Subclonal SCNAs, which are generated by later events during tumorigenesis, play an important role in boosting single-cell heterogeneity. In the mutational scenarios, the ubiquitous mutations are formed early in tumor-initiating cells and are inherited by their offspring, whereas the "private" mutations accumulate sporadically and markedly increase the ITH among different individuals. Subclonal SCNAs and sporadic mutations might impart further advantages to certain subpopulations during tumor growth and mutually facilitate the ITH.

We applied 40 single cells and 48 single cells to evaluate the ITH for patients 1 and 2, respectively. After removing the diploid somatic cells, there were 24 and 35 tumor cells with SCNAs for patients 1 and 2, respectively. A previous study on breast cancer suggested that 20-40 single cells were eligible for detecting SCNA-based subpopulations [13], which was compatible with our results about subclonal SCNAs. Therefore, the amount of single cells for each patient we have studied was reasonable. The computationally derived tumor percentage of each region was determined by Sequenza (Additional file 1: Fig. S3). The separated regions of one tumor were assessed by the pathologists, of which the histological features were reckoned similar. The tumor purifies of two patients identified by the pathologists were both more than $90 \%$, but the deduced results of WES showed that the tumor purity of P1 was just 25-49\% (Additional file 1: Fig. S3) owing to somatic cell infiltration. The lower tumor purity of P1 might give rise to lower ITH in some extent, since the diploid cell contamination would mask the true profiles, distorting the SCNA profiles and descending the mutational heterogeneity by missing low frequency mutations. When obtaining the tumor mutations by WES, the germline mutations could be excluded by comparing tumor regions to peripheral blood or normal rectum samples. Here, we utilized peripheral blood but not normal rectum as control in order to avoid missing somatic mutations that existed early in both adjacent normal tissues and tumors, which is rare but could happen in some cases.

The heterogeneity of distinct regions of one tumor arises from the proportion of various subclones. Tumor tissue is a mixture of different cell populations that interact with the microenvironment, and the evolution of tumorigenicity is complex and dynamic. The preponderant subclone adapting to the circumjacent microenvironment plays a dominant role in certain region of one tumor, of which the master status is dynamically changing. For instance, though substantial tumor cells could be killed during the therapy, there were still survival of rare subclones with resistance to drugs, which might lead to relapse. It is the heterogeneity that make some tumors so hard to eradicate. At single-cell level, SCNAs were confirmed to be in correlation with gene expression [43], and the SCNAs of colorectal cancer, which affected the expression of functional genes, were reported to be potential biomarkers [35]. For instance, there was only one population according to the large-scale copy number profiles in patient 1, but when zoom in to focal SCNA alterations, there were apparently two subpopulations, meaning that although the large-scale copy number profiles (24 chrmosomes) appear to be similar at this time snap-shot, the single tumor cells possibly form two subpopulations owing to the differences in subclonal SCNAs in the future. Besides clonal SCNAs which all tumor cells steadily inherited, subclonal SCNAs would facilitate further cell-to-cell heterogeneity, which might lead to different therapy requirement. Among the subclonal SCNAs in patient 1, MINA, which is located in the focal region chr3q11.2, is a c-Myc target gene that may affect cell proliferation [44]. The tumor suppressor genes PIK3C3 on chr18q12.3 and SMAD2 on chr18q21.1, which affected the TGF- $\beta$ pathway, were reported to be related to metastasis [35, 45]. SCNAs induced upregulation or downregulation of these important genes would eventually give rise to growth advantages in certain populations during tumor progression.

Two patients were of the same age, no smoking, no alcohol intake, and both adenocarcinoma without microsatellite instable. The protein biomarkers of two tumors were different, CEA was highly expressed in P1, while CA72.4 was highly expressed in P2. Even though P2 (T3), which had one lymph node metastasis and positive nerve invasion, was further progressed than P1 (T2), the postoperative therapy was quite effective. The regular follow-up showed that the two patients under personalized medicine were healthy with no relapse after surgery. Consistent with previous studies [46], our study also demonstrated the mutational diversification of multiple regions and branch evolution in rectal cancer. Additionally, we found that the regional differences in SCNA profiles of 
different tumor regions might arise from different subpopulations (Fig. 3a and b). Single-cell sequencing further confirmed the distributions of minor subpopulations, and revealed the subclonal structure of the tumor. Minor cell populations might exist early in tumorigenesis but in limited quantities, or they might be generated later with extraordinary growth advantages [47].

Tumors are composed of many cells, and bulk sequencing only reveals the average genomic alterations of this cell mixture; thus, clonal analysis cannot resolve the subclonal composition of a tumor beyond the resolution of the sample used for the analysis. Contamination by diploid cells and the proportions of tumor subpopulations may affect the SCNA profiles of tumor regions. Moreover, deep sequencing is required to detect rare mutations in bulk tumor, which is costly. Thus, single-cell sequencing is of significant importance in investigating tumor cell heterogeneity and in discovering subtle diversification. However, it should be noted that we did not find any correlation between the copy number variation and mutation events. In accordance with the previous report [48], our results also suggest that a single biopsy is sufficient for determination of major copy number profiles and highfrequency mutations for target therapy, however, it is insufficient for precise detection of subclonal SCNAs and low-frequency mutations.

In a conclusion, although the two patients are of the same molecular classification, the extent of heterogeneity differed. There are different clinicopathological features and molecular paths of tumorigenesis in colon and rectal cancer [3], so it is meaningful to focus just on rectal tumors. Personalized medicine, tailored to each individual based on druggable genes, is necessary. In addition, the extensive ITH might also indicate that there are many possibilities for drug resistance in each patient. This study provides a preliminary impression of ITH in rectal cancer.

\section{Conclusions}

The SCNA profiles of multiple regions and single tumor cells within one tumor are similar, suggesting that a considerable number of SCNAs are early events in cancer development and inherited steadily. The regional differences of SCNA profiles within multiple regions arise from different proportions of SCNA-based subpopulations. Single-cell WGS shows focal SCNAs that were not detected in the multi-region WES, implying that a detailed genetic characterization of the tumor can be better uncovered by single-cell sequencing. Although the two patients are of the same molecular classification, the extent of heterogeneity differed. Intertumor heterogeneity supports the necessary of personalized medicine tailored to each patient based on clonal target genes. Intratumor heterogeneity means there are many possibilities for drug resistance in each patient.

\section{Additional files}

Additional file 1: Figs. S1-S5 and Tables S1-S6. (DOCX 1794 kb)

\begin{abstract}
Abbreviations
COSMIC: Catalog of Somatic Mutations in Cancer; CTCs: Circulating tumor cells; FACS: Fluorescent activated cell sorting; ITH: Intratumor heterogeneity; MAPD: Median of the absolute values of all pairwise differences; PAM: Partition around medoids; PCA: Principle component analysis; SCNAs: Somatic copy number alterations; SNVs: Single nucleotide variations; VAF: Variant allelic frequency; WES: Whole-exome sequencing; WGS: Whole-genome sequencing
\end{abstract}

\begin{abstract}
Acknowledgements
We thank Mr. Zhonglin Fu and Ms. Xuefang Zhang from the National Center for Protein Sciences Beijing (Peking University) for assistance with FACS; Ms. Yu Hou from BIOPIC in Peking University for academic assistance; Dr. Shuang Geng from BIOPIC in Peking University for accurate sorting with FACS; and colleagues in Peking University Cancer Hospital \& Institute for collecting specimens.
\end{abstract}

\section{Funding}

This work was supported by the Peking University (PKU) 985 Special Funding for Collaborative Research with PKU Hospitals (to FB and XS), the National High Technology Research and Development Program of China (863 Program, No. 2015AA020403 to FB), the National Key Research and Development Program (2016YFC0900100 to FB), the Beijing Municipal Science \& Technology Commission (No. Z141100000214013 to FB), and the Recruitment Program of Global Youth Experts (to FB), the National Natural Science Foundation of China (No. 81272766, No. 81450028, and No. 81672439 to XS, and No. 81502137 to JD) on study design, sample collection and preparation, sequencing experiments and data analysis, the Beijing Natural Science Foundation (No. 7162039 to XS) on paper publication, and the Beijing Municipal Administration of Hospitals Clinical Medicine Development of Special Funding Support (No. XM201309 to XS and No. ZYLX201701 to Dr. Jiafu Ji) on team cooperation.

\section{Availability of data and materials}

The raw data in the fastq format of this study was available in the NCBI Sequence Read Archive under the SRA study accession SRP093555.

\section{Authors' contributions}

FB, XS, ML, JD and YL conceived and designed the study; JD, HY and MZ collected samples and patient information, processed tumor tissue and performed FACS; ML did the sequencing experiments; $Y L, M L$ and ZS analyzed and interpreted the data; BJ and ZW were responsible for patient follow-up, technical support regarding experiments and interpreting the results; $M L$ drafted the article; FB, JD and XS revised critically for important intellectual content in the manuscript and provided financial support. All authors read and approved the final manuscript and agreed to be accountable for all aspects of the work.

\section{Ethics approval and consent to participate}

The study was approved by the Research Ethics Committee of Peking University Cancer Hospital \& Institute, Beijing, China (No. 2014KT98). Written informed consent was obtained from two patients for use of these clinical samples for research.

\section{Consent for publication}

Not applicable.

\section{Competing interests}

The authors declare that they have no competing interests.

\section{Publisher's Note}

Springer Nature remains neutral with regard to jurisdictional claims in published maps and institutional affiliations. 
Received: 10 May 2017 Accepted: 13 November 2017

Published online: 23 November 2017

\section{References}

1. Brenner H, Kloor M, Pox CP. Colorectal cancer. Lancet. 2014;383:1490-502.

2. Cancer Genome Atlas Network. Comprehensive molecular characterization of human colon and rectal cancer. Nature. 2012;487:330-7.

3. Tamas K, Walenkamp AM, de Vries EG, van Vugt MA, Beets-Tan RG, van Etten B, et al. Rectal and colon cancer: not just a different anatomic site. Cancer Treat Rev. 2015;41:671-9.

4. Yates LR, Gerstung M, Knappskog S, Desmedt C, Gundem G, Van Loo P, et al. Subclonal diversification of primary breast cancer revealed by multiregion sequencing. Nat Med. 2015;21:751-9.

5. Cao W, Wu W, Yan M, Tian F, Ma C, Zhang Q, et al. Multiple region whole-exome sequencing reveals dramatically evolving intratumor genomic heterogeneity in esophageal squamous cell carcinoma. Oncogene. 2015;4:e175.

6. Gerlinger M, Horswell S, Larkin J, Rowan AJ, Salm MP, Varela I, et al. Genomic architecture and evolution of clear cell renal cell carcinomas defined by multiregion sequencing. Nat Genet. 2014;46:225-33.

7. Gerlinger M, Rowan AJ, Horswell S, Larkin J, Endesfelder D, Gronroos E, et al. Intratumor heterogeneity and branched evolution revealed by multiregion sequencing. N Engl J Med. 2012;366:883-92

8. de Bruin EC, McGranahan N, Mitter R, Salm M, Wedge DC, Yates L, et al. Spatial and temporal diversity in genomic instability processes defines lung cancer evolution. Science. 2014;346:251-6.

9. Zhang J, Fujimoto J, Zhang J, Wedge DC, Song X, Zhang J, et al. Intratumor heterogeneity in localized lung adenocarcinomas delineated by multiregion sequencing. Science. 2014;346:256-9.

10. Ni X, Zhuo M, Su Z, Duan J, Gao Y, Wang Z, et al. Reproducible copy number variation patterns among single circulating tumor cells of lung cancer patients. Proc Natl Acad Sci U S A. 2013;110:21083-8.

11. Navin N, Kendall J, Troge J, Andrews P, Rodgers L, Mclndoo J, et al. Tumour evolution inferred by single-cell sequencing. Nature. 2011:472:90-4.

12. Wang $Y$, Waters J, Leung ML, Unruh A, Roh W, Shi X, et al. Clonal evolution in breast cancer revealed by single nucleus genome sequencing. Nature. 2014,512:155-60

13. Gao R, Davis A, McDonald TO, Sei E, Shi X, Wang Y, et al. Punctuated copy number evolution and clonal stasis in triple-negative breast cancer. Nat Genet. 2016:48:1119-30

14. Yu C, Yu J, Yao X, WSu WKK, Lu Y, Tang S, et al. Discovery of biclonal origin and a novel oncogene SLC12A5 in colon cancer by single-cell sequencing. Cell Res. 2014;24:701-12.

15. Zong C, Lu S, Chapman AR, Xie XS. Genome-wide detection of single-nucleotide and copy-number variations of a single human cell. Science. 2012;338:1622-6.

16. Li H, Durbin R. Fast and accurate short read alignment with burrowswheeler transform. Bioinformatics. 2009;25:1754-60.

17. Li H, Handsaker B, Wysoker A, Fennell T, Ruan J, Homer N, et al. The sequence alignment/map format and SAMtools. Bioinformatics. 2009;25:2078-9.

18. McKenna A, Hanna M, Banks E, Sivachenko A, Cibulskis K, Kernytsky A, et al. The genome analysis toolkit: a MapReduce framework for analyzing next-generation DNA sequencing data. Genome Res. 2010;20:1297-303.

19. Josephidou M, Lynch AG, Tavaré S. multiSNV: a probabilistic approach for improving detection of somatic point mutations from multiple related tumour samples. Nucleic Acids Res. 2015;43:e61.

20. Cingolani P, Platts $A$, Wang le $L$, Coon $M$, Nguyen $T$, Wang $L$, et al. A program for annotating and predicting the effects of single nucleotide polymorphisms, SnpEff: SNPs in the genome of Drosophila Melanogaster strain w1118; iso-2; iso-3. Fly (Austin). 2012;6:80-92.

21. Xue R, Li R, Guo H, Guo L, Su Z, Ni X, et al. Variable intra-tumor genomic heterogeneity of multiple lesions in patients with Hepatocellular carcinoma. Gastroenterology. 2016;150:998-1008.

22. Tamura K, Peterson D, Peterson N, Stecher G, Nei M, Kumar S. MEGA5: molecular evolutionary genetics analysis using maximum likelihood, evolutionary distance, and maximum parsimony methods. Mol Biol Evol. 2011;28:2731-9.

23. Favero F, Joshi T, Marquard AM, Birkbak NJ, Krzystanek M, Li Q, et al. Sequenza: allele-specific copy number and mutation profiles from tumor sequencing data. Ann Oncol. 2015;26:64-70.

24. Cai X, Evrony GD, Lehmann HS, Elhosary PC, Mehta BK, Poduri A, et al. Single-cell, genome-wide sequencing identifies clonal somatic copy-number variation in the human brain. Cell Rep. 2014;8:1280-9.
25. Huang DW, Sherman BT, Lempicki RA. Systematic and integrative analysis of large gene lists using DAVID bioinformatics resources. Nat Protoc. 2008:4:44-57.

26. Huang DW, Sherman BT, Lempicki RA. Bioinformatics enrichment tools: paths toward the comprehensive functional analysis of large gene lists. Nucleic Acids Res. 2009;37:1-13.

27. Vogelstein B, Papadopoulos N, Velculescu VE, Zhou S, Diaz LA Jr, Kinzler KW. Cancer genome landscapes. Science. 2013;339:1546-58.

28. Domingo E, Ramamoorthy R, Oukrif D, Rosmarin D, Presz M, Wang H, et al. Use of multivariate analysis to suggest a new molecular classification of colorectal cancer. J Pathol. 2013;229:441-8.

29. Alexandrov LB, Nik-Zainal S, Wedge DC, Aparicio SA, Behjati S, Biankin AV, et al. Signatures of mutational processes in human cancer. Nature. 2013;500:415-21.

30. Gundem G, Van Loo P, Kremeyer B, Alexandrov LB, Tubio JMC,

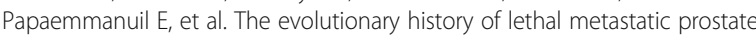
cancer. Nature. 2015;520:353-7.

31. Schell MJ, Yang M, Teer JK, Lo FY, Madan A, Coppola D, et al. A multigene mutation classification of 468 colorectal cancers reveals a prognostic role for APC. Nat Commun. 2016;7:11743.

32. Pectasides $E$, Bass AJ. ERBB2 emerges as a new target for colorectal cancer. Cancer Discovery. 2015;5:799-801.

33. Brown CJ, Lain S, Verma CS, Fersht AR, Lane DP. Awakening guardian angels: drugging the p53 pathway. Nat Rev Cancer. 2009:9:862-73.

34. Pino MS, Chung DC. The chromosomal instability pathway in colon cancer. Gastroenterology. 2010;138:2059-72.

35. Wang H, Liang L, Fang JY, Xu J. Somatic gene copy number alterations in colorectal cancer: new quest for cancer drivers and biomarkers. Oncogene. 2016:35:2011-9.

36. Gosens MJ, van Kempen LC, van de Velde CJ, van Krieken JH, Nagtegaal ID. Loss of membranous Ep-CAM in budding colorectal carcinoma cells. Mod Pathol. 2007;20:221-32.

37. Huang L, Ma F, Chapman A, Lu S, Xie XS. Single-cell whole-genome amplification and sequencing: methodology and applications. Annu Rev Genomics Hum Genet. 2015:16:79-102.

38. Hou Y, Wu K, Shi X, Li F, Song L, Wu H, et al. Comparison of variations detection between whole-genome amplification methods used in singlecell resequencing. Gigascience. 2015;4:37.

39. Reynolds AP, Richards G, de la Iglesia B, Rayward-Smith VJ. Clustering rules: a comparison of partitioning and hierarchical clustering algorithms. Journal of Mathematical Modelling and Algorithms. 2006;5:475-504.

40. Hiley C, de Bruin EC, McGranahan N, Swanton C. Deciphering intratumor heterogeneity and temporal acquisition of driver events to refine precision medicine. Genome Biol. 2014;15:453.

41. Mayans O, van der Ven PFM, Wilm M, Mues A, Young P, Furst DO, et al. Structural basis for activation of the titin kinase domain during myofibrillogenesis. Nature. 1998;395:863-9.

42. Stewart-Hutchinson PJ, Hale CM, Wirtz D, Hodzic D. Structural requirements for the assembly of LINC complexes and their function in cellular mechanical stiffness. Exp Cell Res. 2008:314:1892-905.

43. Macaulay IC, Haerty W, Kumar P, Li YI, TX H, Teng MJ, et al. G\&T-seq: parallel sequencing of single-cell genomes and transcriptomes. Nat Methods. 2015; 12:519-22.

44. Teye K, Tsuneoka M, Arima N, Koda Y, Nakamura Y, Ueta Y, et al. Increased expression of a Myc target gene Mina53 in human colon cancer. Am J Pathol. 2004;164:205-16.

45. González-González M, Fontanillo C, Abad MM, Gutiérrez ML, Mota I, Bengoechea $\mathrm{O}$, et al. Identification of a characteristic copy number alteration profile by high-resolution single nucleotide polymorphism arrays associated with metastatic sporadic colorectal cancer. Cancer. 2014;120: 1948-59.

46. Hardiman KM, Ulintz PJ, Kuick RD, Hovelson DH, Gates CM, Bhasi A, et al. Intra-tumor genetic heterogeneity in rectal cancer. Lab Investig. 2016;96:4-15.

47. Burrell RA, McGranahan N, Bartek J, Swanton C. The causes and consequences of genetic heterogeneity in cancer evolution. Nature. 2013; 501:338-45.

48. Bettoni F, Masotti C, Habr-Gama A, Correa BR, Gama-Rodrigues J, Vianna MR, et al. Intratumoral genetic heterogeneity in rectal cancer: are single biopsies representative of the entirety of the tumor? Ann Surg. 2017;265:e4-6. 